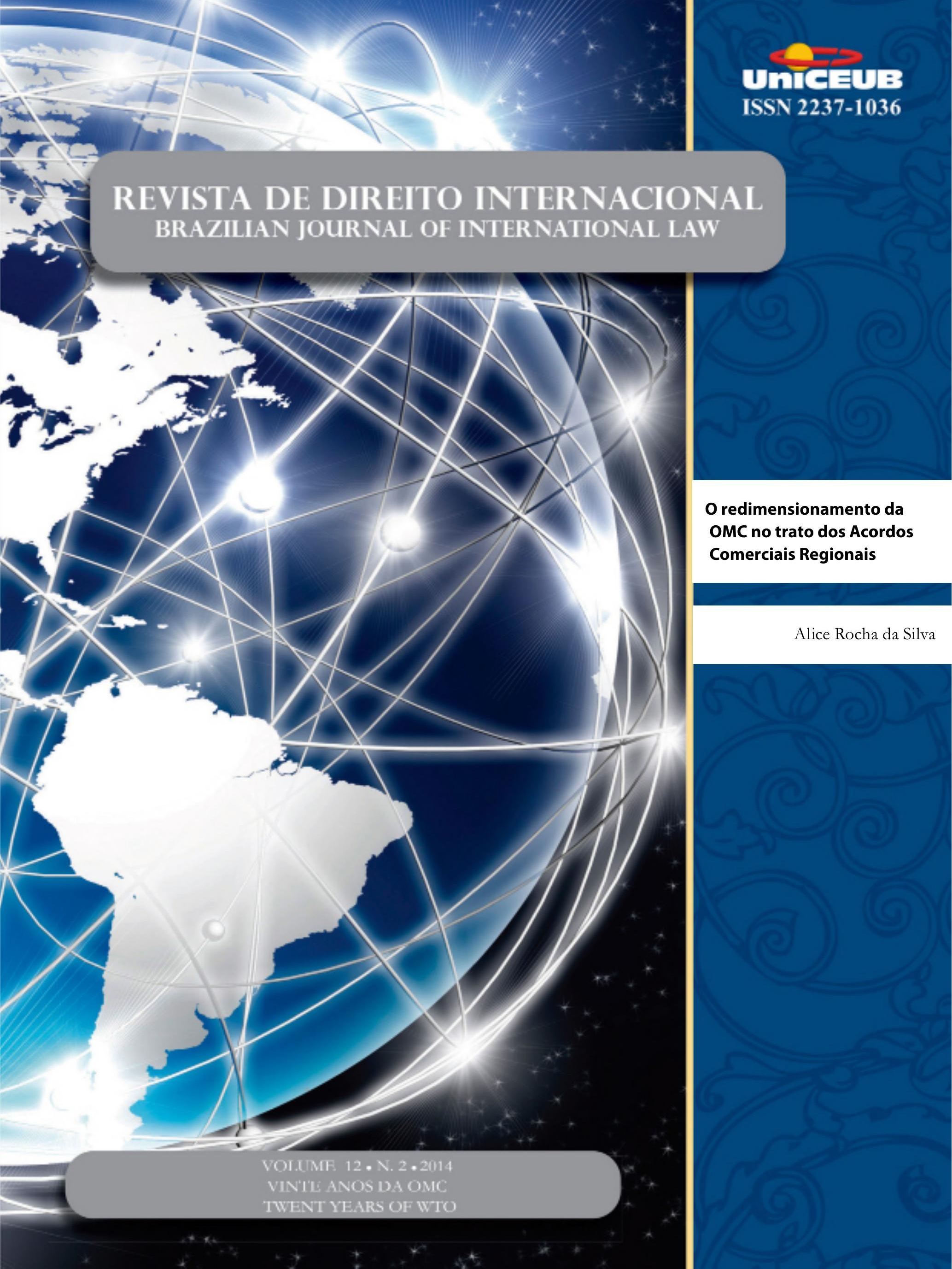


I. Crônicas

1. Crônicas da atualidade do Direito Internacional ......................................................16

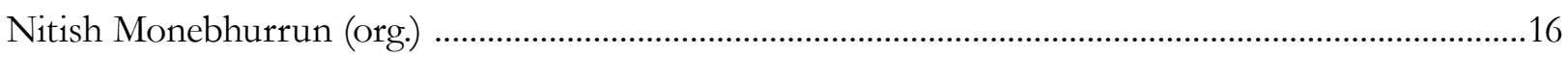

2.Decisões da Corte Internacional de Justiça e do Tribunal Internacional Sobre o Direito do Mar

Nitish Monebhurrun

José Eduardo Siqueira

3. Crônicas do direito internacional dos inVestimentos

Nitish Monebhurrun

\section{Os Vinte Anos dA OMC}

EXPORT CONTROLS AS INDUSTRIAL POLICY ON NATURAL RESOURCES: REGULATORY LIMITATIONS ON CHINA - RAW MATERIALS AND CHINA - RARE EARTHS CASES.

Gustavo Ferreira Ribeiro

O problema da espionagem econômica internacional: Seria a Organização Mundial do CoMÉRCIO O FORO ADEQUADO PARA SUA APRECIAÇÃO?

Humberto A.Vasconcelos Lima

Naiana Magrini Rodrigues Cunha

International Standards for Intellectual Property Rights Protection: a reflection on CLIMATE-FRIENDLY TECHNOLOGY TRANSFER.

Guihong Zhang

Jiani Jiang

Can Wang

Os vinte anos da OMC, suas conquistas e desafios: uma análise do Brasil e o Sistema de SoLUÇõES DE CONTROvÉRsias

Etiene M. Bosco Breviglieri

Luciano Meneguetti Pereira

A relação entre os tratados multilaterais ambientais e os acordos da OMC: é possível CONCILIAR O CONFLITO?

Fabio Costa Morosini,

Luisa Zuardi Niencheski 
Um desafio na Organização Mundial do Comércio: viabilidade de um aCordo plurilateral SOBRE ENERGIA

Matheus Linck Bassani

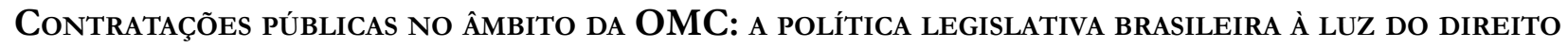
AO DESENVOLVIMENTO

André Jansen do Nascimento

GovernanÇa global e a Organização Mundial do Comércio: desafios impostos pelo novo MANDATO DE DESENVOLVIMENTO

Letícia de Souza Daibert

Ana Luísa Soares Peres

Vinte Anos de Crise para a África? Poder, Assimetrias e a Abordagem Liberal da OMC.....239 Igor Abdalla Medina de Souza

Os MECANISMOS DE INDUÇÃo AO CUMPRIMENTO NO ÂMBITO DA OMC 258

Fernando Lopes Ferraz Elias

A promoção de accountability na Organização Mundial do Comércio: uma análise horiZONTAL E VERTICAL

Celso Henrique Cadete de Figueiredo

LA OMC Y EL PROCESO DE GLOBALIZACION DE LA REGULACIÓN ALIMENTARIA

Maria Eugenia Marichal

O ACORDO GATS E SUA APLICAÇÃo AOS SERVIÇOS DO COMÉRCIO ELETRÔNICO

Gleisse Ribeiro Alves

A OMC E o REgionalismo do SÉculo XXI: ESTRATÉGIA DE IMPOSIÇÃo DE MODELOS NORMATIVOS? .. 337

Camilla Capucio

A organizaÇão mundial do Comércio e a ChINa: direito de Propriedade E PRopriedade INTELECTUAL NO PAÍS.

Dos contenciosos na OMC com ENFoque EM REstrições Às EXPORTAÇões da China .363

Marco Antônio Alcântara Nascimento 
O Redimensionamento da OMC no trato dos Acordos Comerciais Regionais.

Alice Rocha da Silva

III. Outros Temas

Derechos Humanos EN LA REALIDAD ACTUAL: LA GLOBALIZACión Y EL MULTICULTURALISMo

David Falcão

IMUNIDADE DE JURISDIÇÃo do ESTAdo E REPARAÇÃo CIVIL PELA PRÁtICA DE TORTURA: O CASO ZaHRA

KaZEMI v. REPÚblica IsLÂMICA do IRÃ

Patrícia Maria Lara Abreu

Rodrigo Otávio Bastos Silva Raposo

INTERREGIONAL ORgaNIZATIONS (IROS) IN EUROPE: NEW SUBJECTS OF CONTEMPORARY INTERNATIONAL LAW?

Davorin Lapas

A CONEXÃo ENTRE OS DiREITOS HUMANOS E A CORRUPÇÃo

Gabriela Alves Mendes Vieira

Marcelo Dias Varella

GRUPO DE SOCIEDADES: INSTRUMENTO JURÍDICO DE ORGANIZAÇÃo DA EMPRESA PLURISSOCIETÁRIA......495

Daniel Amin Ferraz 


\title{
O redimensionamento da OMC no trato dos Acordos Comerciais Regionais*
}

\section{Redimensioning the WTO in Regional Trade Agreements}

\author{
Alice Rocha da Silva**
}

\section{Resumo}

A complexa articulação entre os acordos comerciais regionais e o direito da OMC pode ser facilitada a partir de instrumentos jurídicos presentes na teoria do Direito Internacional Público. A prática corrente demonstra a possibilidade de sucesso na utilização dessas ferramentas. No entanto, mesmo que tal utilização se revele fraca ou ineficaz, este trabalho visa proporcionar pistas de reflexão para a solução da interação entre regionalismo e multilateralismo. A ação em duas frentes (normativa e jurisdicional) parece permitir uma análise do conjunto que pode resultar em uma melhor interação entre eles. Nesse sentido, começamos nosso trabalho, analisando o fortalecimento do regionalismo comercial e, em seguida, verificaremos os efeitos desse fenômeno sobre o multilateralismo da OMC. A partir da análise proposta, podemos constatar que atualmente os acordos comerciais regionais constituem um elemento essencial na vida comercial dos Estados em todo o mundo e continuarão a coexistir com o sistema da OMC. Assim, a melhor solução seria a de aceitá-los não como um obstáculo aos objetivos da OMC, mas como uma alternativa dada aos Estados para fortalecer ainda mais a liberalização do comércio e encontrar a melhor maneira de articular as relações entre eles no contexto internacional.

Palavras-chave: Articulação. Acordos comerciais regionais. Direito da OMC.

\section{Abstract}

The complex articulation between regional trade agreements and WTO law can be facilitated from legal instruments present in the theory of public international law. The practice demonstrates the possibility of success in using these tools. However, even if such use proves weak or ineffective, this study aims to provide food for thought for the solution of the interaction between regionalism and multilateralism. The action on two fronts (normative and jurisdictional) seems to permit an analysis of the joint which can result in better interaction between them. In this sense, we start our work by analyzing the strengthening of trade regionalism and then see the effects of this phenomenon on the multilateralism of the WTO. From the proposed analysis, we note that currently the regional trade agreements are a key element in the commercial life of states around the world and will continue to coexist with the WTO system. Thus, the best solution would be to not accept them as an obstacle to the objectives of the WTO, but as an alternative given to states to further strengthen trade liberalization and find the best way to articulate the relationship between them in the international context.

Keywords: Articulation. Regional trade agreements. WTO law.

\footnotetext{
* Recebido em: 10.12 .2014

Aceito em: 15.12.2014

** Professora do Programa de Mestrado e Doutorado em Direito do Centro Universitário de Brasília (UniCEUB) e da graduação da Faculdade Processus. Doutora em Direito Internacional Econômico pela Aix-Marseille Université, França. Mestre em Direito das Relações Internacionais pelo UniCEUB. Graduada em Direito pelo UniCEUB e Graduada em Relações Internacionais e Ciência Política pela Universidade de Brasília-UnB. Email: rochaalice@ yahoo.com.br.
} 


\section{Introdução}

Partindo da premissa de que o direito da OMC autoriza e limita a formação de acordos comerciais regionais (ACR), constata-se que, apesar dessas limitações, nas últimas duas décadas, o sistema jurídico internacional tem conhecido uma verdadeira proliferação de ACR. Relações cada vez mais complexas entre regionalismo e multilateralismo são então estabelecidas, sendo necessário o desenvolvimento de instrumentos jurídicos que contribuam para a compreensão da articulação entre os ACR e o direito da OMC.

O direito da OMC oferece aos seus Membros a possibilidade de celebrar ACR, ou seja, permite a formação de tais acordos, desde que preencham os critérios determinados em seus dispositivos legais. Esses critérios são utilizados para limitar os efeitos de desvio de comércio que podem ser causados pela formação de acordos regionais. Vale ressaltar que, desde o início, “[...] o verdadeiro objetivo do GATT era de controlar e limitar a discriminação, não de eliminá-la."1

Nesse sentido, a formação de ACR, entre os Membros da OMC, é possível, mas limitada pelos critérios e procedimentos projetados especificamente para essa finalidade. Esses critérios estão previstos essencialmente no artigo XXIV do GATT, mas também no artigo V do Acordo Geral sobre Comércio de Serviços (GATS do inglês General Agreement on Trade in Services) para tratar os ACR que afetam o comércio de serviços e na Cláusula de Habilitação ${ }^{2}$ para os ACR compostos por países em desenvolvimento, que têm direito a um tratamento diferenciado e mais favorável.

No entanto, as limitações impostas pela OMC para a formação desses ACR não são efetivas e as tentativas de análise de compatibilidade dos ACR com esses critérios foram abandonados pelo Comitê de Acordos Comerciais Regionais (CRTA do inglês Committee on Regional Trade Agreements) que preferiram adotar uma estratégia que favorece uma maior transparência sobre tais acordos ${ }^{3}$. Além disso, se a compatibilidade dos ACR

1 HUDEC, R. E. The Gatt legal system and world trade diplomacy. Salem: Butterworth Legal Publishers, 1990.

2 GATT, Traitement Difference et Plus Favorable Reciprocite et Participation Plus Complete des Pays en Voie de Developpement: Décision du 28 novembre 1979 (Clause d'habilitation), L/4903, 03 décembre 1979.

3 OMC. Mécanisme pour la transparence des accords pudesse eventualmente ser examinada pela Orgão de Solução de Controvércias (OSC) da $\mathrm{OMC}^{4}$, na prática, esse exame seria improvável de acontecer e os Estados temeriam que essas práticas discriminatórias no contexto dos ACR fossem colocadas em questão pelos demais Membros da OMC. Assim, as dificuldades na aplicação desses limites impostos pela OMC, associadas às constantes dificuldades de negociações no campo multilateral, levaram os Estados a tomarem um atalho utilizando a via regional para a defesa de seus interesses.

Nesse sentido, percebe-se que a capacidade operacional da OMC em controlar tais acordos é questionada não só pelas relações de poder e assimetria que sempre existiram, mas também pelas novas dimensões de governança internacional, com redes de regulação e coordenação entre políticas públicas nacionais e globais. A proliferação dos ACR significa a multiplicação desses espaços regulatórios que muitas vezes vão além dos temas tratados pela $\mathrm{OMC}$, podendo ser uma perspectiva de oportunidade para novos negócios, mas também de conflito aos interesses do sistema multilateral. Sendo assim, podemos propor uma avaliação do papel da OMC para a governança internacionals dessas interelações que se formam entre o regionalismo e o multilateralismo, apontando instrumentos jurídicos de articulação entre ambos.

\section{Estabelecimento de precisões conceituais}

Primeiramente, temos de apresentar algumas definições utilizadas na presente análise. Este estudo define o "direito da OMC", como sendo, de um lado, o conjunto de regras contidas no Acordo constitutivo da OMC e seus anexos 1, 2 e 3, que são obrigatórios para todos os Membros da organização, assim como o anexo 4 (acordos comerciais plurilaterais), obrigatórios unicamente para os Membros que os ratificaram. O Acordo Geral sobre Tarifas Aduaneiras e Comércio de 1994, conforme especificado no Anexo 1A (GATT 1994), é um acordo legalmente separado do Acordo Geral sobre Tarifas e Comércio de 1947 (GATT 1947), que também deve

commerciaux régionaux. Décision du 14 décembre 2006, WT/L/671, 18 décembre 2006.

4 A competência do OSC para aplicar os dispositivos ligados ao regionalismo foi estabelecida pelo artigo 3 do Memorando do acordo para solução de controvérsias (MASC) e pelo parágrafo 12 do Memorando do acordo sobre o artigo XXIV, que enuncia que os dispositivos de solução de controvérsias são susceptíveis de serem utilizados na contestação de questões ligadas a aplicação das regras da OMC relativas ao tratamento do regionalismo. 
ser considerado como parte deste conjunto de regras chamadas "direito da OMC". O "direito da OMC" deve, contudo, ser distinguidos dos "acordos abrangidos", tendo em vista o fato de que estes restringem o campo de aplicação do Memorando do acordo para solução de controvérsias (MASC).

Da mesma forma, no contexto desta análise, os ACR devem ser entendidos como acordos internacionais celebrados por escrito entre sujeitos de direito internacional e destinados a produzir efeitos jurídicos no direito internacional. Ou seja, os ACR se incluem na categoria de tratados regidos pela Convenção de Viena sobre o Direito dos Tratados de 1969, que prevê no seu art. 2 par. 1 que "tratado" significa um "acordo internacional concluído por escrito entre Estados e regido pelo Direito Internacional, quer conste de um instrumento único, quer de dois ou mais instrumentos conexos, qualquer que seja sua denominação específica.".

No entanto, esses acordos internacionais possuem duas particularidades. Por um lado, são acordos comerciais, sendo concluídos no contexto das trocas comerciais entre os Estados, no domínio de bens, serviços e propriedade intelectual. A essa particularidade material deve ser acrescida sua especificidade especial: esses acordos se diferenciam dos acordos comerciais estabelecidos no âmbito da OMC pelo seu caráter regional, visto que ligam dois (maioria dos ACR) ou apenas alguns Estados.

Esse caráter regional, no sentido estrito do termo, tornou-se insuficiente para definir os ACR. De fato, muitos ACR foram concluídos entre Estados de uma mesma região ou continente, mas a participação de membros que não são vizinhos imediatos aumentou consideravelmente. Os ACR "transatlânticos" e "transpacíficos" estão se tornando cada vez mais frequentes, como por exemplo os acordos de livre comércio entre a Associação Europeia de Livre Comércio (EFTA) - Chile e o acordo Coreia-EUA. ${ }^{8}$

5 Os "acordos abrangidos" estão enumerados no Apêndice 1 do Memorando do acordo para solução de controvérsias.

6 "The rules and procedures of this Understanding shall apply to disputes brought pursuant to the consultation and dispute settlement provisions of the agreements listed in Appendix 1 to this Understanding (referred to in this Understanding as the "covered agreements")."

7 Convention de Vienne sur le droit des traités, faite à Vienne, 23 mai 1969, 1155 R.T.N.U. 331, 8 International Legal Materials 679.

8 "Traditionally, RTA formation occurred between so-called "natural" trading partners, geographically contiguous countries with already well-established trading patterns. [...] Indeed, most countries
Embora a expressão "acordo comercial regional" não seja a mais adequada para o tratamento de diversos acordos que não estão limitados a Estados de uma mesma região, ela continua sendo utilizada no contexto dessa análise, e isso não só porque é a mais empregada pela OMC e pela doutrina, mas também porque, atualmente, não é possível examinar as questões entre bilateralismo e multilateralismo deixando de lado acordos que ultrapassam o quadro regional. De forma mais precisa, a caracterização destes acordos em ACR é justificada pelo fato de que eles mantêm a característica principal dos ACR, ou seja, o aspecto discriminatório em relação aos demais Membros da OMC: esses acordos consistem em exceções ao princípio da nação mais favorecida. Note-se também que esta análise se concentra apenas em acordos preferenciais recíprocos — regionais, bilaterais ou plurilaterais ${ }^{9}$ — uma vez que representam a maioria dos ACR, visto que quase 90 por cento da margem total de preferência correspondem a tarifas preferenciais aplicadas no contexto de acordos recíprocos. ${ }^{10}$

Deve ser ainda definida a noção de "jurisdição" ou de "mecanismo jurisdicional" que no contexto de nossa análise serão entendidos em sentido amplo, abrangendo tribunais permanentes, mas também os tribunais arbitrais ad hoc, previstos na maioria dos ACR. Além disso, esse conceito de jurisdição inclui também as soluções negociadas com a valorização dos aspectos políticos do caso.

sign their first RTA with one or several neighbouring or regional partners. [...] However, once a country has exhausted its strictly regional prospects, it may begin to look further afield for preferential partners." FIORENTINO, R. V.; VERDEJA, L.; TOQUEBOEUF, C. Discussion Paper n. 12: The changing landscape of regional trade agreements 2006 update. p. 12. Disponível em: <http://www.wto.org/english/res_e/ publications_e/disc_paper12_e.htm>. Acesso em: 10 dez. 2014.

9 Em resumo, a diferença entre os acordos bilaterais e plurilaterais está no número de participantes: os bilaterais comportam duas partes e os plurilaterais mais de dois. Entretanto, os acordos bilaterais podem ser constituídos por vários Estados organizados em grupos, como no caso dos ACR compostos por ACR preexistentes. Esta situação parece ser a nova tendência no regionalismo. Alguns exemplos de ACR com tais características concluídos ou em negociação são UE-MERCOSUR, UE-CCG, União Aduaneira da Africa Austral (SACU). GONCALVES, J. B. Livre-comércio ou comércio mais livre? alternativas ao multilateralismo. In: LUZ, M. (Coord). Comércio e negociacões internacionais para jornalistas. Centro Brasileiro de Relações Internacionais, 2009. p. 43-47.

10 Ver OMC. Rapport sur le commerce mondial 2011. L'OMC et les accords commerciaux préférentiels: de la coexistence à la cohérence. p. 72-85. Disponível em: <http://www.wto.org/french/res_f/ publications_f/wtr11_f.htm>. Acesso em: 27 nov. 2014. 
Essa definição flexível do conceito de jurisdição internacional é essencial, visto que, se a natureza jurisdicional do Orgão de Solução de Controvérsias da OMC pode ser questionada, o caráter jurisdicional dos mecanismos dos ACR seria ainda mais questionável. No entanto, mesmo considerando-se que os painéis da OMC seriam uma representação do Conselho Geral, que deve representar os Membros da $\mathrm{OMC}^{11}$ e não um verdadeiro órgão jurisdicional, é preciso considerar que o Órgão de Apelação comporta diversos elementos que o aproxima da noção de jurisdição internacional: composto por membros permanentes, fornece considerações de base jurídica e limitado às questões de direito incluídas no relatório do painel. No entanto, deve ser considerado que os relatórios dos painéis e do Órgão de Apelação não são vinculantes. A aquisição de sua força obrigatória está sujeita à aprovação do relatório pelo Órgão de Solução de Controvérsias. Se esta característica do sistema de solução de controvérsias da OMC pudesse ser um fator decisivo na rejeição da sua qualificação como jurisdição internacional, torna-se indispensável salientar que a aprovação do relatório se dá por consenso negativo. ${ }^{12}$ Logo, na prática, a possibilidade de não se adotar um relatório do Painel e do Órgão de Apelação é quase nula, e por esta razão é possível considerar que a adoção desses relatórios é quase automática. ${ }^{13}$

Estabelecidos tais conceitos operacionais, partiremos para a análise da evolução do regionalismo comercial em paralelo ao sistema multilateral da OMC.

\section{$3 \mathrm{O}$ fortalecimento do regionalismo comercial}

O fenômeno do regionalismo ganhou importância em todo o mundo, não só quantitativamente, mas também qualitativamente. Motivações políticas são subjacentes à formação desses acordos. Desde muito tempo, a redução tarifária ligada aos acordos preferenciais tem sido considerada a principal razão para sua formação. Chegou-se a pensar que, com as reduções oferecidas ao final da Rodada Uruguai, a tendência de participação em ACR seria enfraquecida, o que não

11 "The General Council shall convene as appropriate to discharge the responsibilities of the Dispute Settlement Body provided for in the Dispute Settlement Understanding. [...]" Acordo de Marrakech instituindo a OMC, art. IV: 3.

12 Art. 16 et 17:14 du MASC.

13 No mesmo sentido: ROMANO, C.P.R. The proliferation of international judicial bodies: the pieces of the puzzle. N.Y.U. Journal of International Law and Politics, v. 31, n. 4, p. 709-751, 1999. at. p. 719. ocorreu. Em vez disso, os ACR proliferaram-se de forma ainda mais vigorosa. Podemos, a esse respeito, verificar a complexidade das razões que levam os Estados a optarem por um ou outro sistema, regional ou multilateral. Afinal, devem ser considerados os elementos de ordem política e estratégica, que demonstram que os ACR vão muito além da mera liberalização tarifária. ${ }^{14}$

A formação dos ACR foi a estratégia utilizada pelos Estados Unidos para estabelecer boas relações com o Oriente Médio após os ataques terroristas de 11 de setembro de 2001. Esse país procurou associar, na formação de acordos preferenciais, interesses políticoestratégicos e comerciais, e torná-los uma forma de promover os interesses e parcerias estratégicas em todo o mundo. Quando o acordo entre os Estados Unidos e o Marrocos foi assinado, o representante do comércio norte-americano, Robert Zoellick declarou que: “[s]tep by step, the Administration is working to build bridges of free trade with economic and social reformers in the Middle East. Our plans offer trade and openness as vital tools for leaders striving to build more open, optimistic and tolerant Islamic societies." ${ }^{15}$

A quantidade de ACR aumentou e a taxa do comércio mundial, regido por esses acordos, é cada vez mais importante. No entanto, atualmente, os Estados estão mais interessados na expansão do comércio do que no número de acordos regionais em vigor. Nesse sentido, o que é importante na análise do regionalismo, não é apenas o número de ACR estabelecidos, mas também o volume de comércio que efetivamente acontece no contexto desses acordos.

Em relação ao volume de comércio representado pelos ACR, a OMC não controla esses dados e há uma certa divergência entre os dados fornecidos por diferentes fontes externas a OMC, tais como relatórios e pesquisas de outras organizações internacionais. Pascal Lamy, diretor-geral da OMC, declarou em 2006 que as estatísticas concluíram que tal volume representava um percentual superior a $50 \%$ do comércio mundial. ${ }^{16}$ Essa previsão também foi discutida

14 No mesmo sentido, VAN DEN BOSSCHE, P. The law and policy of the World Trade Organization: text, cases and materials. 2 ed. Cambridge: Cambridge University Press, 2008. p. 697.

15 United States. United States Trade Representative, Press Release, 15 June 2004. United States and Marocco sign historic free trade agreement. Disponível em: <www.ustr.gov> Acesso em: 03 out. 2014.

16 OMC, Nouvelles 2006, M. Lamy salue l'accord de l'OMC sur les accords commerciaux régionaux, ler juillet 2006. 
em estudo de 2003 da Organização para a Cooperação e Desenvolvimento Econômico (OCDE).${ }^{17}$ No entanto, esse percentual equivale ao volume de comércio movimentado pelos ACR em geral, o que não significa que são resultantes das regras preferenciais em relação às taxas estabelecidas na OMC. Além disso, deve ser considerado que muitas tarifas aplicadas em ACR já são consolidadas a zero na OMC. Isso significa que o comércio feito com base nessas taxas não cria preferências para parceiros regionais e, portanto, perde todo o caráter discriminatório em termos de comércio mundial. ${ }^{18} \mathrm{O}$ Banco Mundial também conduziu um estudo que estimou que, em 2004/2005, 30 a 35\% do comércio internacional foi efetivamente praticado no contexto dos ACR. Se for desconsiderado o comércio com base em tarifas consolidadas a zero no sistema da OMC, a percentagem de comércio realmente discriminatório cai para um valor de $21 \%$ do comércio mundial. ${ }^{19}$ Além disso, o relatório da OMC de 2011 confirma esses dados, fornecendo informações que mostram que a participação do comércio intra-ACR no comércio mundial passou de 18\% em 1990 para 35\% em $2008^{20}$. Os dados relacionados ao comércio de serviços intra-ACR também são limitados porque poucos Estados comunicam às organizações internacionais as estatísticas sobre esta categoria de comércio, e não há, no momento, uma base de dados detalhada e padronizada sobre este tema. ${ }^{2}$

17 ORGANISATION for economic co-operation and development. Regionalism and the Multilateral Trading System. Paris: OECD, 2003. p. 12.

18 Esta constatação é confirmada pelo relatório de 2011 da OMC:"si le nombre d'ACPr a augmenté, l'importance du commerce préférentiel n'a pas suivi. Cette évolution résulte d'une forte réduction des droits NPF au cours des deux dernières décennies, soit dans le cadre de négociations commerciales multilatérales soit sur une base unilatérale." OMC, Rapport sur le commerce mondial 2011. L'OMC et les accords commerciaux préférentiels : de la coexistence à la cohérence, p. 72. Disponível em: <http://www.wto.org/french/res_f/ publications_f/wtr11_f.htm>. Acesso em: 27 set. 2014.

19 WORLD Bank, Global economic prospects. Washingon: World Bank, 2005. p. 40-41.

20 OMC. Rapport sur le commerce mondial 2011. L'OMC et les accords commerciaux préférentiels: de la coexistence à la cobérence, p. 64. Disponível em: <http://www.wto.org/french/res_f/publications_f/wtr11_f. htm>. Acesso em: 27 set. 2014.

21 Para maiores informações sobre os dados relativos aos principais exportadores e importadores de serviços (UE e Estados Unidos) ver OMC. Rapport sur le commerce mondial 2011. L'OMC et les accords commerciaux préférentiels : de la coexistence à la cobérence. $\mathrm{p}$. 67-69. Disponível em: <http://www.wto.org/french/res_f/ publications_f/wtr11_f.htm>. Acesso em: 27 set. 2014.
Se nos apoiarmos nos dados da OMC, especialmente na notificação de novos ACR e adesões a ACR existentes, notaremos que até o dia 15 de junho de 2014, 585 ACR (separando as notificações relativas a bens e notificações relativas a serviços) foram notificados ao GATT / OMC. Destes, 391 ACR estão atualmente em vigor, 230 foram notificados nos termos do artigo XXIV do GATT, 37 sob a Cláusula de Habilitação e 124 nos termos do artigo $\mathrm{V}$ do GATS. ${ }^{22}$

Ora, mais de 90\% dos ACR notificados e em vigor consistem em zonas de livre comércio (ZLC) ou acordos de alcance parcial (o termo Inglês 'partial scope'), enquanto menos de $10 \%$ dos ACR notificados são representados por uniões aduaneiras (UA). Quanto aos ACR que ainda não tenham sido notificados ou que estão em fase de negociação ou de proposição, a preferência por ZLC e por acordos parciais é confirmada.

Comparando-se as notificações feitas no âmbito do GAT'T, com as notificações no âmbito da OMC, podese notar um aumento considerável das notificações na OMC em comparação com as realizadas no GATT. ${ }^{23}$ Esse aumento pode ser explicado pela entrada de novos Membros na OMC e pelo reforço da obrigação de notificação no caso do comércio de serviços. No entanto, a metodologia adotada pela OMC tem falhas e nem sempre produz dados que refletem a realidade.

A metodologia utilizada pela OMC não reflete o número real de ACR em vigor, visto que os ACR abrangendo serviços e bens são notificados de forma independente, mesmo que em alguns casos correspondam ao mesmo acordo. Além disso, a adesão de novos membros à ACR existentes é considerada como um novo acordo, prática que pode ser amplamente questionada. Por outro lado, a contabilidade do número "real" de ACR também pode ser questionada, uma vez que não há nenhuma maneira de verificar os dados de ACR que ainda não foram notificados ou que estão em etapas de implementação diferentes. Apesar dessas

22 BASE de données sur les ACR. Disponível em: $<$ http://rtais.wto.org/UI/publicsummarytable.aspx $>$. Acesso em: 02 nov. 2014.

23 A taxa anual de notificação nda OMC é de 20, enquanto no quadro do GATT era de somente 3. Em quase 50 anos de existência do GATT 1947, 124 notificações relativas a ACR foram recebidas, ao passo que, em 20 anos, a OMC já recebeu mais de 300 notificações incluindo comércio de bens e serviços. Ver GHÉRARI, H. Le bilatéralisme conquérant ou le nouveau visage du commerce international. $R G D I P$, n. 2, p. 255-293, 2008. 
limitações, a metodologia adotada pela OMC ainda é considerada a mais adequada para demonstrar um fenômeno sem precedentes: a proliferação de acordos comerciais regionais.

Atualmente, apenas alguns dos 160 membros da OMC não fazem parte de um ACR: Mongólia, Djibouti, Mauritânia, Sudão do Sul, Somália e República Democrática do Congo. Os dados apresentados pelo Comitê de Acordos Regionais (CRTA) em 2000 mostram que, em média, cada um dos Membros do sistema multilateral de comércio está ligado a cinco acordos preferenciais e alguns estão envolvidos em mais de dez acordos. ${ }^{24}$ No caso da União Europeia, a tarifa consolidada na OMC a partir do tratamento da nação mais favorecida se aplica de modo integral a somente seis membros do sistema multilateral. Para os outros parceiros, que ultrapassam uma centena, a União Europeia prevê um tratamento mais favorável do que o previsto pela OMC. ${ }^{25}$

A partir desses dados quantitativos e qualitativos, verifica-se a emergência de uma nova onda de regionalismo que se opõe ao fenômeno constatado pela primeira vez na década de $60 .{ }^{26} \mathrm{O}$ "novo regionalismo" se caracteriza não só pelo forte aumento do número de ACR, mas também por uma nova dimensão espacial, que inclui novos parceiros, incluindo ACR já existentes e novos domínios materiais.

De fato, os novos ACR contêm disposições que abrangem outras áreas. Além do comércio de bens e serviços, esses ACR apresentam disposições relativas a

24 OMC. Comité des accords commerciaux régionaux, Synopsis des "questions systémiques" relatives aux accords commerciaux régionaux, WT/REG/W/37, 2 mars 2000, p. 4. O Chile é um dos membros mais ativos na OMC, participando de mais de 16 ACR ao mesmo tempo. Base de dados sobre os ACR disponível em: $<$ http://rtais.wto.org/UI/PublicMaintainRTAHome.aspx $>$. Os Estados Unidos também utilizam bastante a via bilateral. Para mais detalhes ver o site oficial do United States Trade Representative (USTR): < http://www.ustr.gov/trade-agreements/ free-trade-agreements>. Acesso em: 04 set. 2014.

25 Comentários sobre tais dados são apresentados em: BHAGWATI, J.N. Termites in the trading system: how preferential agreements undermine free trade. Oxford: Oxford University Press, 2008. SAPIR, A. The political economy of EC regionalism. European Economic Review, v. 42, p. 717-732, 1998. WTO, CONSULTATIVE Board to the WTO Director-General, The future of the WTO: advancing institutional challenges in the new Millenium. Geneva: WTO, 2004. p. 21.

26 WHALLEY, J. Recent regional agreements: why so many, why so much variance in form, why coming so fast, and where are they headed? The World Economy, v. 31, n. 4, p. 517-532, 2008. Pour une analyse de l'implication des États-Unis dans le nouveau régionalisme voy. HILAIRE, A; YANG, Y. The United States and the new regionalism/ bilateralism. IMF Working Paper, WP/03/206, 2003. 25 p. investimento, a normas técnicas, a propriedade intelectual, a regras de concorrência, de trabalho e de meio ambiente, algumas vezes visando à inclusão destes na OMC. Mesmo os ACR considerados pouco aprofundados no campo da cooperação, como as zonas de livre comércio, começam a abordar questões como os contratos públicos, investimento, defesa comercial, entre outros. Essa nova tendência de incluir medidas que geralmente não são cobertas pela OMC é chamada de "regionalismo agressivo", sendo o acordo de livre comércio entre a Coreia e os Estados Unidos um bom exemplo. ${ }^{27}$

Além da proliferação quantitativa dos ACR, a observação empírica do regionalismo mostra que os Membros da OMC preferem integrações econômicas menos aprofundadas. Além disso, traços desse novo regionalismo são as novas formas híbridas de integração, os novos domínios incluídos nos ACR, o aumento do número de acordos Norte-Sul e a sobreposição de ACR, além da conclusão de acordos entre dois ACR préexistentes ou entre países geograficamente distantes. Deve ser ressaltado, que, juntamente com a proliferação de novos ACR, em geral, eles possuem seus próprios mecanismos de solução de controvérsia que também apresentam uma proliferação sem precedente.

\section{A proliferação de mecanismos de solução de controvérsias previstos em ACR}

Paralelamente a essa proliferação substancial dos ACR, constata-se que o Órgão de Solução de Controvérsias da OMC está cada vez mais confrontado a proliferação de mecanismos regionais para a solução de controvérsias previstos em ACR. A multiplicação de fóruns internacionais de resolução de controvérsias parte de uma atribuição progressiva de capacidades, contribuindo para o crescimento da complexidade no sistema jurídico internacional e do risco de que tal sistema torne-se cada vez mais fragmentado, incoerente e não sistêmico. ${ }^{28}$ Assim, a proliferação de fóruns regionais, em concorrência ou não com o direito da OMC, chamou a atenção para os perigos da fragmentação do sistema jurídico internacional e para

27 Para uma análise desta nova tendência ver CHOI, W. M. Aggressive regionalism in Korea-US FTA: the present and future of Korea's FTA policy. Journal of international economic law, v. 12, n. 3, p. 595-615, 2009.

28 VARELLA, M. D. A crescente complexidade do sistema jurídico internacional: alguns problemas de coerência sistêmica. Revista de Informação Legislativa, Brasília, v. 42, n. 167, p. 163, jul/set. 2005. 
a necessidade de instrumentos que possam prevenir e tratar possíveis conflitos resultantes da ação paralela destes fóruns internacionais. Os Estados parte nos ACR e Membros da OMC são obrigados a respeitar os dois acordos e a utilização de um fórum regional pode acarretar em violações das regras da OMC e vice-versa.

Decisões conflitantes surgem do fato que vários acordos são aplicados simultaneamente. De fato, não existe uma regra de direito internacional que define uma proibição de recursos paralelos aos dois sistemas, acumulando procedimentos e formando situações de litispendência, na qual um único litígio é submetido a ambas as jurisdições. A análise dos dispositivos da OMC mostra que seus Membros não perceberam o potencial de conflitos passíveis de surgimento entre as jurisdições regionais e o Órgão de Solução de Controvérsias (OSC) da OMC. Como exemplo, o artigo XXIV do GATT, o artigo $\mathrm{V}$ do GATS e a Cláusula de Habilitação não possuem nenhum dispositivo ou limite a incorporação de mecanismos regionais de resolução de litígios nos ACR.

A motivação para a criação de fóruns regionais pode ser explicada pelas lacunas em fóruns universais, no tratamento de questões precisas e específicas do contexto regional. No caso do direito internacional econômico, a criação de fóruns regionais é fundamental porque alguns ACR se concentram em questões que a OMC não trata, o que tem implicações em termos de resolução de disputas. Nesse sentido, os sistemas de resolução de conflitos regionais são necessários, uma vez que são as únicas jurisdições competentes para o tratamento de determinados assuntos.

Alguns mecanismos de resolução de litígios regionais são semelhantes ao OSC da $\mathrm{OMC}^{29}$, como o mecanismo previsto no âmbito do Acordo de Livre Comércio da América do Norte (NAFTA). Entre essas semelhanças, constata-se a pertinência dada a inclusão de cláusulas de eleição de forum no caso de "forum shopping". A prática do fórum shopping ocorre quando mais de uma jurisdição terá a competência para tratar do caso e as partes podem escolher o foro para lidar com o caso. ${ }^{30}$ No entanto, mesmo com essas cláusulas,

29 No mesmo sentido ver GHÉRARI, H. La concurrence des procédures dans le contentieux de l'OMC. In: KERBRAT, Y. Forum Shopping et concurrence des procédures contentieuses internationals. Bruxelles: Bruylant Bruxelles, 2011, p. 77-102. at. p. 82.

30 Existem diversas definicoes para o termo: "Forumshopping: the practice of introducing a proposal or pursuing a dispute in one forum after another until a favourable outcome has been achieved. Sometimes also called forum-shopping." GOODE, W. Dictionary of trade policy terms. 5. ed. WTO : Cambridge, 2007. p. as partes ainda têm a possibilidade de um eventual fórum shopping e um paralelismo de procedimentos que podem resultar em decisões diferentes e até mesmo contrárias geradas por fóruns autônomos. Nessa situação, deve ser verificada a importância dada aos aspectos políticos para a resolução de casos, visto que podem ser decisivos para a escolha da decisão a ser aplicada: processos paralelos e decisões contraditórias podem existir e são admitidas pelo direito internacional.

\section{Os efeitos da proliferação de ACR para o sistema multilateral de comércio da OMC}

A partir desse contexto podemos constatar que a complexidade do sistema internacional se deve a três fatores diferentes: a expansão normativa e a acumulação de normas; o aumento de regimes especiais e a consequente diversificação do direito internacional e da jurisdicionalização desse direito. ${ }^{32}$ Assim, verificase a importância de se analisar a relação entre o sistema multilateral da OMC com o ACR, no sentido de que a criação dos ACR constitui uma violação dos princípios fundamentais da OMC, especialmente o princípio da nação mais favorecida e que a proliferação inesperada desses acordos pode prejudicar a legitimidade da OMC na liderança da liberalização do comércio mundial.

De fato, ACR estão se multiplicando a uma velocidade inesperada, e em alguns casos, eles

179 "Forum-shopping: (terme anglais, sans équivalent en français - Voy. cependant le français québécois «magasinen»: choisir un tribunal comme on choisit d'entrer dans une boutique pour faire ses emplettes): possibilité qu'offre à un demandeur la diversité des règles de compétence internationale de saisir les tribunaux du pays appelé à rendre la décision la plus favorable à ses intérêts.» CORNU, G. Vocabulaire juridique. 8. ed. Paris:PUF, 2004. p. 397. "Forumshopping: the practice of choosing the most favorable jurisdiction or court in which a claim might be heard.” BLACK, H.C. Black's law dictionary: definitions of the terms and phrases of American and English jurisprudence, ancient and modern. St. Paul, Minn.: West Publishing Co., 1990.

31 SANTULLI, C. Droit $d u$ contentieux international. Paris: Montchrestien, 2005. p. 91.

32 GHÉRARI, H. La concurrence des procédures dans le contentieux de l'OMC. In: KERBRAT, Y. Forum Shopping et concurrence des procédures contentieuses internationals. Bruxelles: Bruylant Bruxelles, 2011. p. 77-102. at. p. 78- 79.

33 Um estudo sobre os riscos da interação entre os ACR e o sistema multilateral de comércio da OMC e as possíveis soluções para as relações estabelecidas entre eles foi desenvolvido em TRAN, T.T.P. Les accords régionaux dans le système de l'Organisation mondiale $d u$ commerce. 2007. 534 f. Thése (doctorat) Université Panthéon-Assas. Paris, 2007. 
são considerados uma ameaça ao multilateralismo estabelecido pela OMC. A aproximação entre os Estados Unidos e a União Europeia na construção de uma parceria transatlântica tem sido apontada como um risco potencial para o sistema da OMC. Embora ainda não exista um acordo preferencial entre eles, desde a década de 1990, foram tomadas iniciativas para promover o aumento do comércio e dos investimentos entre as duas entidades. Em 2004, foi adotado um documento chamado 'EU-US Initiative to Enhance Trans-Atlantic Economic Integration and Growth', o qual indica claramente a intenção de liberalizar o comércio entre as duas regiões. ${ }^{34}$

Se um acordo de livre comércio entre eles se materializasse, haveria um impacto significativo sobre o multilateralismo, pois, juntos, respondem por $40 \%$ do volume do comércio mundial e aproximadamente $60 \%$ do PIB mundial. A formação de um acordo de livrecomércio entre os Estados Unidos e a União Europeia comporta, de fato, riscos para o sistema multilateral da OMC, porque se for bem-sucedido, a OMC passaria a ser um brinquedo nas mãos dos Estados Unidos e da União Europeia, servindo somente para dar legitimidade às decisões tomadas no contexto bilateral. No entanto, o sistema multilateral não pode ser substituído por tais acordos e é por isso que um equilíbrio entre os dois polos deve ser procurado. ${ }^{35} \mathrm{Na}$ mensagem do director geral da OMC, Roberto Azevedo, no relatório anual da OMC de 2014, ele deixou bem claro que tais acordos possuem um papel de complementação ao sistema multilateral, mas que não seriam suficientes em si por excluir diversos atores econômicos e diversas "grandes questões", como por exemplo as reduções de subsídios agrícolas, só seriam passíveis de tratamento ao nível mundial. ${ }^{36}$

No entanto, devemos também levar em conta o fato de que, durante a negociação de acordos comerciais regionais, existe a possibilidade de que alguns temas

34 Sobre esse tema ver: SILVA, A. R. Crônicas de Direito Internacional: acordo de livre-comércio transatlântico Estados Unidos-União. Revista de Direito Internacional, v. 10, n.1, 2013.

35 Este foi o título do discurso do Diretor Geral da OMC, Roberto Azevedo no enceramento do Seminário da OMC sobre as questões transversais nos ACR, no dia 25 de setembro de 2014. Disponível em: <http://www.wto.org/french/news_f/spra_f/ spra33_f.htm> Acesso em: 02 nov. 2014.

36 OMC. Rapport annuel 2014. Disponível em: <http://www. wto.org/french/res_f/booksp_f/anrep_f/anrep14_f.pdf $>$. Acesso em: 10 dez. 2014. sejam melhor tratados e esta discussão aprofundada sobre determinados domínios pode ser posteriormente introduzida nas regras multilaterais. Resistências para o aprofundamento do tratamento de determinados assuntos no sistema da OMC podem ser anuladas a nível regional. Além disso, a abertura dos mercados por meio dos ACR se revela menos traumática para os países em desenvolvimento, dada a possibilidade de uma exposição gradual da economia nacional à concorrência externa.

Tópicos tratados superficialmente ou simplesmente ignorados pelo sistema multilateral da OMC estão presentes nos acordos regionais. Isso pode ser explicado pela dificuldade das negociações a nível multilateral, tendo em vista a maior quantidade de Membros e interesse em jogo. A prova disso é que na atual Rodada Doha, as questões que não estão previstas no mandato original da Rodada são eliminadas. Com efeito, a conclusão dessa rodada tornou-se uma prioridade para a Organização e o tratamento destas questões poderia retardar ainda mais o período de negociações para a conclusão da Rodada a partir do estabelecimento de um engajamento único. ${ }^{37}$

Ao estabelecer o mandato da Rodada de Doha, houve uma tentativa de incluir temas como os contratos de investimento e de compras governamentais, mas depois disso eles foram explicitamente excluídos da agenda de negociações. Isso incluiu a questão da facilitação do comércio, que ainda está em negociação. ${ }^{38}$ Outros temas de interesse para as empresas multinacionais, como as regras relativas à governança, corrupção, responsabilidade social e imigração nem sequer são mencionados no sistema multilateral. Isso incentiva os operadores públicos e privados a encontrarem outras instâncias para o tratamento destas questões. Assim, as questões não abrangidas pela OMC estão cada vez mais integradas ao nível regional. Pode-se desta forma considerar a intenção de abordar estas questões no quadro multilateral no futuro.

Em particular, temas sensíveis como as normas de trabalho, de meio ambiente e de propriedade intelectual

37 Diversas sugestões relativas a reformas no processo de negociação da OMC foram apresentadas, entre elas a reavaliação do « engajamento único » ('single undertaking') que pode bloquear as negociações. Para análise das proposições de reforma ver ROLLAND, S.E. Redesigning the negotiation process at the WTO. Journal of International economic Law, v. 13, n. 1, p. 65-110, 2010.

38 OMC. Programme de travail de Doha, Décision adoptée par le Conseil général le 1er août 2004. WT/L/579. 02 août 2004. 
são atualmente tratadas principalmente nos ACR formados pelos principais atores dentro do sistema, dado o maior poder de barganha que eles possuem nesse universo mais restrito. Assim, alguns assuntos estão agora entrando por uma "porta lateral" no sistema da OMC. ${ }^{39}$

Se os ACR podem ser considerados uma ameaça para o sistema da OMC, eles também podem contribuir para o fortalecimento do multilateralismo. Uma verdadeira sinergia entre as duas abordagens pode ocorrer, como no caso do Memorando do GATS sobre compromissos relativos a serviços financeiros, estabelecido com auxílio da experiência adquirida no contexto da abertura dos mercados financeiros no nível regional. No entanto, essa interação nem sempre é fácil, uma vez que a OMC e os acordos comerciais regionais não são elementos estáticos. Considere o fato de que os ACR crescem e evoluem, não apenas na base de regras multilaterais, mas também em função de outros ACR criados. Além disso, as motivações para a criação de compromissos não são as mesmas, a nível regional e no sistema multilateral, visto que no sistema regional o grau de afinidade entre um número menor de parceiros é maior.

Os ACR podem ajudar a liberalização do comércio, afinal os novos compromissos assumidos em sua esfera podem incentivar a abertura de mercados no nível multilateral. A esse argumento soma-se a "teoria da liberalização competitiva", associada à interação positiva entre as duas dinâmicas, regional e multilateral, desencadeada por negociações simultâneas. A liberalização do comércio em ambas as esferas estabelece uma competição entre elas, o que favorece o multilateralismo comercial, na medida em que ele vai tentar expandir no nível multilateral a liberalização realizada a nível regional. Historicamente, a formação de ACR e a liberalização alcançada dentro deles sempre motivou os Estados para iniciar novas rodadas de negociação no âmbito multilateral. ${ }^{40}$

Associada à teoria da liberalização competitiva, a “teoria do dominó” também procura explicar a interação entre as negociações em ambos os níveis. Inicialmente, essa teoria foi usada para explicar como a experiência

39 SUTHERLAND, P. L'avenir de l'OMC: relever les défis institutionnels du nouveau millénaire. Rapport du Conseil consultatif à M. Supachai Panitchpakdi, Directeur Général, OMC. Genève, 2004. p. 23.

40 BERGSTEN, C.F. Fifty years of the GATT/WTO: lessons from the past for strategies for the future. In: WTO Secretariat, From the GATT to the WTO: the multilateral trading system in the new millennium, The Hague, Kluwer/WTO. 2000. regional bem-sucedida incentivou a formação de novos acordos comerciais regionais ou expansão dos ACR existentes. Em uma segunda etapa, essa teoria tem sido utilizada para demonstrar a interação positiva que se desenvolve entre o regionalismo e o sistema da OMC, e foi ainda mais longe ao afirmar que, mesmo que a relação não ocorra de forma muito ordenada e fora do controle da OMC, a formação de ACR contribui para a evolução do sistema multilateral como um todo. ${ }^{41}$

Esse efeito dominó funciona em ambas as direções, a partir do multilateralismo para o regionalismo ou do regionalismo para o multilateralismo. Isso quer dizer que, da mesma forma que as disciplinas negociadas no contexto multilateral influenciam as negociações regionais, as regionais influenciam nas negociações multilaterais. Esse fenômeno foi facilmente percebido no setor de serviços, porque as negociações realizadas por meio de listas positivas de serviços, assim como a definição dos modos de obrigação foram lições aprendidas com o acordo de livre-comércio entre o Canadá e os Estados Unidos (em inglês CanadaUnited States Free Trade Agreement - CUSFTA) e foram refletidas no texto do GATS. Outro exemplo pode ser apreendido com o NAFTA que se inspirou da experiência do GATS e foi além substituindo a lista positiva pela lista negativa, acentuando a liberalização. ${ }^{42}$

Tal teoria deve ser aplicada com cautela e sem generalização sobre a automaticidade dos acontecimentos. Novos estudos empíricos precisam abordar estes quesitos, porque a prática das negociações de serviços da Rodada de Doha tem mostrado o contrário: os países mais aplicados no tratamento desta disciplina a nível regional, como os Estados Unidos, são mais reticentes nas negociações multilaterais, o que parece contradizer a teoria do dominó. ${ }^{43}$ Assim, parece que o jogo de dominós é mais acessível aos principais atores que têm a capacidade de fazer com que a dinâmica regional influencie positivamente o multilateralismo, mas nesse contexto deve ser questionado se estes atores

41 Para análise desta teoria sob o ponto de vista econômico ver BALDWIN, R. Multilateralising regionalism: spaghetti bowls as building blocs on the path to global free trade, Geneva: Institute for International Studies, 2006. p. 15 e ss.

42 Para uma análise mais aprofundada sobre este exemplo ver OSTRY, S. Regional dominoes and the WTO: building blocks or boomerang? Fraser Institute Conference. Toronto: [s.n.], nov. 1999. p. 05.

43 ROY, M.; MARCHETTI, J.; LIM, H. Services liberalization in the new generation of preferential trade agreements (PTAs): how much further than the GATS? WTO Staff Working Paper. Geneva: WTO, 2006. p. 58. 
estão dispostos a pagar o preço para apoiar o sistema da OMC, em detrimento dos ganhos regionais.

Os Estados têm se aproveitado de experiências regionais para o tratamento de vários temas na OMC, mas deve-se ressaltar que o processo não é automático nem linear, afinal existem diversos outros elementos que são essenciais para as negociações a nível multilateral. Basta ter em mente que os Estados podem concordar em participar de ACR que tratem de questões negligenciadas pela $\mathrm{OMC}$ e se recusam a incluir na agenda de negociações multilaterais.

O regionalismo deve ser visto como uma ferramenta e não como um fim em si, devendo ser utilizado para o desenvolvimento do comércio entre os parceiros regionais. ${ }^{44} \mathrm{Um}$ fator positivo é o caráter inclusivo de alguns ACR, que buscam se ampliar através da adesão de novos membros e da inclusão de novos objetos, ao invés de se fechar em si mesmos. ${ }^{45}$ Aliados a esses fatores de proliferação e articulação entre regionalismo e multilateralismo, resta interessante apresentar uma análise prospectiva sobre o papel da OMC e do multilateralismo para a governança mundial aliada a novos arranjos regionais.

\section{O papel da OMC e do multilateralismo para a governança mundial aliada a novos arranjos regionais}

Estudos recentes da OMC demonstram que a tendência de proliferação dos ACR constatada nos últimos anos pode sofrer uma "reversão". Isso não significa que os ACR irão desaparecer e sim que a parte do comércio realizada em tais acordos poderá sofrer uma diminuição. ${ }^{46}$

44 Para a análise desse ponto de vista no contexto do Mercosul ver: KLOR, A.D. O jogo de luzes e sombras do Mercosul. Pontes, v. 5, n. 3, out 2009. Disponível em: < http://ictsd.net/i/news/ pontes/53552/>. Acesso em: 28 out. 2014.

45 Ver por exemplo as boas práticas desenvolvidas no contexto da APEC: MARCEAU, G. The adoption of the 'best practice' for regional and free trade agreements in APEC: a road towards more WTO-consistent regional trade agreements. In: TANIGUCHI, Y.; YANOVICH, A.; BOHANES, J. (Ed.). The WTO in the Twenty-first Century: dispute settlement, negotiations, and regionalism in Asia. Cambridge: Cambridge University Press, 2006. 507 p. at p. 409-422.

46 FONTAGNÉ, Lionel; FOURÉ, Jean; KECK, Alexander. Simulating world trade in the decades ahead: driving forces and policy implications, 2014. Disponível: <http://www.wto.org/english/ res_e/reser_e/ersd201405_e.pdf >.Acesso em: 10 dez. 2014.
Tal estudo deve ser visto com bastante cuidado, sobretudo no aspecto interpretativo de tal "reversão". Isso porque ele traz uma análise prospectiva da economia mundial em 2035, a partir de técnicas e estudos econômicos, demonstrando que a probabilidade será de que os países se reagrupem em blocos amplos, chamados "megaregionais" sugerindo que tais blocos tenham o viés multilateral e não regional.

Ora, se os países estarão reagrupados nesses megablocos, isso pode significar um risco ainda maior para a pertinência da OMC, visto que uma das razões de sua permanência frente a essa proliferação de ACR é justamente a de que ainda não surgiram ACR entre países desenvolvidos, que precisariam do contexto da OMC para concluírem suas negociações.

O estudo ainda indica a necessidade de que a OMC propicie um ambiente econômico dinâmico e aberto à participação de novos atores, propiciando um incremento no comércio entre os países em desenvolvimento e apoiando tais economias para atividades que exigirão maior intensidade de competências.

Isso constitui um dado bastante relevante para a discussão do papel da OMC na governabilidade internacional, visto que essa organização mostrou-se favorável a ações de apoio aos países desenvolvidos, sendo a própria Rodada Doha voltada para a questão do desenvolvimento. Sendo assim, a OMC se mostrará essencial para coordenar as ações de políticas públicas nacionais e internacionais envolvendo países em desenvolvimento, incluindo os novos atores públicos e privados na implementação de ações voltadas para a inserção destes nesse novo cenário.

A probabilidade é de que o progresso tecnológico representará o maior impacto para a conjectura da economia futura, sendo que aspectos relacionados a fatores populacionais também serão fortemente influenciados. Para alguns países, será indispensável que melhorem a competência de seus trabalhadores, enquanto outros suprirão tais falhas com a imigração de mão de obra especializada.

Enfim, a OMC deverá estar preparada para auxiliar seus membros, principalmente os países em desenvolvimento, na aquisição dos meios para competirem nesse mercado internacional. Não só no apoio a blocos os envolvendo, mas também no auxílio direto a suas políticas econômicas e de regulação com o comércio internacional. 


\section{Conclusão}

A partir dessa constatação de uma pluralidade de fontes e de sistemas de solução de controvérsias, devem ser buscados meios capazes de "ordenar o múltiplo" para "garantir a compatibilidade de normas com proveniência diversa". ${ }^{47}$ Para alguns, deve ser reconhecido o pluralismo do direito internacional por meio do uso de "forças imaginativas do direito" não significa necessariamente a "descrição verdadeira e completa da realidade", pois não se limita à aplicação de técnicas jurídicas e princípios comuns existentes no direito internacional. ${ }^{49}$ Isso não impede uma busca pragmática de regras e princípios jurídicos que possam servir de referencial para o processo de integração normativa.

Nessa perspectiva de "pluralismo ordenado" 50 , propõe-se a articulação entre os ACR e o direito da OMC que não se limita apenas à procura de compatibilidade normativa entre eles, mas também à análise das interações desses acordos, do processo de criação de seus dispositivos e dos mecanismos de monitoramento capazes de tornar previsíveis as variáveis resultantes da aplicação da norma. Além disso, essa noção de pluralismo ordenado refere-se a uma ideia de coordenação e não de subordinação, que é compatível com o cenário de interdependência entre esses acordos. ${ }^{51}$

47 CHEVAlLIER, J. Mondialisation du droit ou droit de la mondialisation. In: MORAND, C. A. (Dir.). Le droit saisi par la mondialisation. Bruxelles: Bruylant 2001. p.135.

48 Expressão empregada por Mirelle Delmas-Marty in DELMAS-MARTY, M. Les forces imaginantes du droit (II): Le pluralisme ordonné. Paris: Editions du Seuil, 2006.

49 JOUANNET, E. A quoi sert le droit international ? Le droit international providence du XXIème siècle. In: RUIZ FABRI, H.; JOUANNET, E.; TOMKIEWICZ, V. (Dir). Select proceedings of the european society of international law. Porteland Oregon: Hart Publishing, 2008. p. 4. v. 1.

50 Um estudo aprofundado sobre a ideia do pluralismo ordenado é desenvolvido por Mireille Delmas-Marty. DELMAS-MARTY, M. Les forces imaginantes du droit: Le pluralisme ordonné. Paris: Editions du Seuil, 2006. v. 2.

$51[\ldots]$ le pluralisme n'est que la suite logique de l'histoire du droit international si l'on admet que ce mouvement signifie non seulement admettre les différences, mais aussi les concilier, voire les ordonner, sans les "écraser"” SOREL, J.M. Le rôle du droit international dans le développement du pluralisme (et vice versa): une liaison moins naturelle qu'il n'y parait. In: FONTAINE, Lauréline (Dir). Droit et pluralisme. Bruxelles: Bruylant, 2007. p. 78.
Para isso, a articulação dos ACR com o direito da OMC pode ser realizada por meio da utilização cruzada e recíproca de seus dispositivos em seus respectivos processos de interpretação, bem como o uso de remédios gerais relevantes do direito processual. As disposições previstas na Convenção de Viena de 1969 podem servir de base para o estabelecimento de certos instrumentos propostos para a articulação normativa entre os ACR e o direito da OMC.

No entanto, além das hipóteses de concorrência e de conflito normativo, também devem ser considerados, para a análise da articulação entre os ACR e o direito da OMC, situações de concorrência entre procedimentos contenciosos internacionais que são o produto de estratégias desenvolvidas pelas partes. Algumas delas podem preferir procedimentos conduzidos em paralelo para aumentar suas chances de sucesso e ao mesmo tempo forçar o réu a desistir. Por sua vez, o réu também pode acionar um outro tribunal para tentar obter uma decisão a seu favor. Essa sobreposição de procedimentos pode criar um risco de insegurança jurídica em casos onde decisões contraditórias são oferecidas sobre a mesma medida, com base em regras muito semelhantes.

Os remédios processuais como a incompetência, a coordenação normativa e a consolidação de procedimentos são importantes porque a partir deles, um dos juízes leva em conta a decisão do outro e contribui para a coordenação entre as instâncias. ${ }^{52}$

A "articulação" deve ser entendida a partir de uma perspectiva de harmonização do direito internacional, onde não é mais possível tratar os conjuntos normativos em um aspecto único, e isso porque cada um desses conjuntos tem suas próprias especificidades. Assim, o mais importante está em procurar estabelecer um quadro normativo internacional onde esses diferentes sistemas normativos possam coexistir de forma coerente. Isso porque, no contexto em que o direito internacional contemporâneo se desenvolve, o processo de integração normativa deve ser percebido como o resultado de diversas forças "entre unidade e pluralidade, entre universalismo e cultura, entre mundialização e regionalização, constitucionalização e fragmentação, entre o direito dos Estados e o direito dos indivíduos, entre o direito dos agentes públicos e

52 Nesse sentido ver RÉMY, B. La concurrence des procédures États-investisseurs. In : KERBRAT, Y. Forum Shopping et concurrence des procédures contentieuses internationales, Bruxelles : Bruylant Bruxelles, 2011. p. 15 a 76. spéc. p. 76. 
o direito dos atores privados." ${ }^{53}$ Além disso, devemos reconhecer o direito internacional como uma ordem jurídica que se realiza por meio de "acumulação". ${ }^{54}$

Atualmente os ACR constituem um elemento indispensável na vida comercial dos Estados do mundo inteiro e eles não estão em processo de desaparecimento. Assim, a melhor solução seria a de aceitá-los não como um obstáculo para os objetivos da OMC, mas como uma alternativa dada aos Estados para fortalecer ainda mais a liberalização do comércio e encontrar a melhor

53 Tradução livre. JOUANNET, E. Le même et l'autre. In: FABRI, Hélène Ruiz; SOREL, Jean-Marc; JOUANNET, Emmanuelle (Dir). Regards d'une génération sur le droit international. Paris: Pedone, 2008. p. 223.

54 Nesse sentido ver REUTER, P. Introduction an droit des traités, Paris: PUF, Publications de l'IUHEI, 1985. p. 110. maneira de articular as relações entre eles no contexto internacional.

Vale ressaltar que ao longo de seus vinte anos de existência, a OMC apresentou progressos no tratamento da questão, ao estabelecer meios mais efetivos de supervisão de tais acordos. Todavia, novos desafios surgem nesse cenário cada vez mais globalizado, onde percebe-se o enfraquecimento do Estado como principal sujeito de direito internacional pela participação de outros atores públicos e privados. No cenário de governança apresentado, juntamente com a perspectiva dos megablocos, a OMC deve ser redimensionada no sentido de contornar possíveis falhas que atingirão, sobretudo os países em desenvolvimento. 


\section{Referências}

ACCORD de libre-échange entre les États-Unis et l'Australie, signé le 18 mai 2004, date d'entrée en vigueur le 1er janvier 2005. Disponível em: <http://www.ustr.gov/tradeagreements/free-trade-agreements/australian-fta/ final-text>. Acesso em: 08 fev. 2014.

BALDWIN, R. Multilateralising regionalism: spaghetti bowls as building blocs on the path to global free trade. Geneva: Institute for International Studies, 2006.

BASE de données sur les ACR. Disponível em: < http:// rtais.wto.org/UI/publicsummarytable.aspx $>$. Acesso em: 10 out 2014.

BERGSTEN, C.F. Fifty years of the GATT/WTO: lessons from the past for strategies for the future. In: WTO Secretariat, From the GATT to the WTO: the multilateral trading system in the new millennium, The Hague, Kluwer/WTO. 2000.

BHAGWATI, J.N. Termites in the trading system: how preferential agreements undermine free trade. Oxford: Oxford University Press, 2008.

BLACK, H.C. Black's law dictionary: definitions of the terms and phrases of American and English jurisprudence, ancient and modern. St. Paul, Minn.: West Publishing Co., 1990.

CHEVALLIER, J. Mondialisation du droit ou droit de la mondialisation. In: MORAND, C. A. (Dir.). Le droit saisi par la mondialisation. Bruxelles: Bruylant 2001.

CHOI, W. M. Aggressive regionalism in Korea-US FTA: the present and future of Korea's FTA policy. Journal of international economic law, v. 12, n. 3, 2009.

CONVENTION de Vienne sur le droit des traités, faite à Vienne, 23 mai 1969, 1155 R.T.N.U. 331, 8 International Legal Materials 679.

CORNU, G. Vocabulaire juridique. 8. ed. Paris:PUF, 2004.

DELMAS-MARTY, M. Les forces imaginantes du droit (II): Le pluralisme ordonné. Paris: Editions du Seuil, 2006.

FIORENTINO, R.V; VERDEJA, L.; TOQUEBOEUF,C. Discussion Paper $n .12$ : the changing landscape of regional trade agreements 2006 update. p.12. Disponível em: <http://www.wto.org/english/ res_e/publications_e/disc_paper12_e.htm $>$ Acesso em: 14 abr. 2014.

GATT, Traitement Difference et Plus Favorable Reciprocite et Participation Plus Complete des Pays en Voie de Developpement : Décision du 28 novembre 1979 (Clause d'habilitation), L/4903, 03 décembre 1979.

GHÉRARI, H. La concurrence des procédures dans le contentieux de l'OMC. In: KERBRAT, Y. Forum Shopping et concurrence des procédures contentieuses internationals. Bruxelles: Bruylant Bruxelles, 2011. p. $77-102$.

GHÉRARI, H. Le bilatéralisme conquérant ou le nouveau visage du commerce international. RGDIP, n. 2, p. 255293, 2008.

GONCALVES, J. B. Livre-comércio ou comércio mais livre? alternativas ao multilateralismo. In: LUZ, M. (Coord). Comércio e negociacões internacionais para jornalistas. Centro Brasileiro de Relações Internacionais, 2009.

GOODE, W. Dictionary of trade policy terms. 5. ed. Cambridge: WTO, 2007.

HILAIRE, A; YANG, Y. The United States and the new regionalism/bilateralism. IMF Working Paper, WP/03/206, 2003.

HINDLEY, B. New institutions for Transatlantic Trade? International Affairs, v. 75, n. 1, p. 45-60, 1999.

HUDEC, R. E. The Gatt legal system and world trade diplomacy. Salem: Butterworth Legal Publishers, 1990.

JOUANNET, E. A quoi sert le droit international? Le droit international providence du XXIème siècle. In: RUIZ FABRI, H.; JOUANNET, E.; TOMKIEWICZ, V. (Dir). Select proceedings of the european society of international law. Porteland Oregon: Hart Publishing, 2008. v. I

JOUANNET, E. Le même et l'autre. In: FABRI, Hélène Ruiz; SOREL, Jean-Marc; JOUANNET, Emmanuelle (Dir). Regards d'une génération sur le droit international. Paris: Pedone, 2008. 
KLOR, A. D. O jogo de luzes e sombras do Mercosul. Pontes, v. 5, n. 3, out 2009. Disponível em: <http:// ictsd.net/i/news/pontes/53552/>. Acesso em: 28 out. 2014.

MARCEAU, G. The adoption of the 'best practice' for regional and free trade agreements in APEC: a road towards more WTO-consistent regional trade agreements. In: TANIGUCHI, Y.; YANOVICH, A.; BOHANES, J. (Ed.). The WTO in the Twenty-first Century: dispute settlement, negotiations, and regionalism in asia. Cambridge: Cambridge University Press, 2006. 507 p., at pp. 409-422.

OMC. Comité des accords commerciaux régionaux, Synopsis des "questions systémiques" relatives aux accords commerciaux régionaux, WT/REG/W/37, 2 mars 2000 .

OMC. Mécanisme pour la transparence des accords commerciaux régionaux. Décision du 14 décembre 2006, WT/L/671, 18 décembre 2006.

OMC. Nouvelles 2006, M. Lamy salue l'accord de l'OMC sur les accords commerciaux régionaux, 1er juillet 2006.

OMC. Programme de travail de Doha, Décision adoptée par le Conseil général le 1er août 2004. WT/L/579. 02 août 2004.

OMC. Rapport sur le commerce mondial 2011. L'OMC et les accords commerciaux préférentiels: de la coexistence à la cohérence, p. 72-85. Disponível em: <http://www. wto.org/french/res_f/publications_f/wtr11_f.htm>. Acesso em: 27 out. 2014.

OMC. Rapport sur le commerce mondial 2011. L'OMC et les accords commerciaux préférentiels : de la coexistence à la cohérence. p. 72. Disponível em: <http://www.wto.org/ french/res_f/publications_f/wtr11_f.htm>. Acesso em: 27 out. 2014.

OMC. Rapport sur le commerce mondial 2011. L'OMC et les accords commerciaux préférentiels: de la coexistence à la cohérence. p. 64. Disponível em: <http://www.wto.org/ french/res_f/publications_f/wtr11_f.htm>. Acesso em: 27 out. 2014.

ORGANISATION for economic co-operation and development. Regionalism and the Multilateral Trading System. Paris: OECD, 2003.
OSTRY, S. Regional dominoes and the WTO: building blocks or boomerang? Fraser Institute Conference. Toronto: [s.n.], nov. 1999.

RÉMY, B. La concurrence des procédures États: investisseurs. In: KERBRAT, Y. Forum Shopping et concurrence des procédures contentieuses internationals. Bruxelles: Bruylant, Bruxelles. 2011. p. 15 a 76.

REUTER, P. Introduction an droit des traits. Paris: PUF Publications de l'IUHEI, 1985.

ROLLAND, S. E. Redesigning the negotiation process at the WTO. Journal of International economic Law, v. 13, n. 1, p. 65-110, 2010.

ROMANO, C.P.R. The proliferation of international judicial bodies: the pieces of the puzzle. N.Y.U. Journal of International Law and Politics, v. 31, n. 4, 1999.

ROY, M.; MARCHETTI, J.; LIM, H. Services liberalization in the new generation of preferential trade agreements (PTAs): how much further than the GATS? WTO Staff Working Paper. Geneva: WTO, 2006.

SANTULLI, C. Droit du contentieux international. Paris: Montchrestien, 2005.

SAPIR, A. The political economy of EC regionalism. European Economic Review, v. 42, 1998.

L'UNITED STATES TRADE REPRESENTATIVE (USTR).[Home page]. Disponível em: <http://www.ustr. gov/trade-agreements/free-trade-agreements $>$. Acesso em: 04 fev. 2014.

SOREL, J.M. Le rôle du droit international dans le développement du pluralisme (et vice versa): une liaison moins naturelle qu'il n'y parait. In: FONTAINE, Lauréline (Dir). Droit et pluralisme. Bruxelles: Bruylant, 2007.

SUTHERLAND, P. Lavenir de l'OMC: relever les défis institutionnels du nouveau millénaire. Rapport du Conseil consultatif à M. Supachai Panitchpakdi, Directeur Général, OMC. Genève, 2004.

TRAN, T.T.P. Les accords régionaux dans le système de l'Organisation mondiale du commerce. 2007.534 f. Thése (doctorat) Université Panthéon-Assas. Paris, 2007 
UNITEDSTATES. United States Trade Representative, Press Release, 15 June 2004. United States and Marocco sign historic free trade agreement. Disponível em: <www. ustr.gov>. Acesso em: 03 out. 2014.

VAN DEN BOSSCHE, P. The law and policy of the World Trade Organization: text, cases and materials. 2. ed. Cambridge: Cambridge University Press, 2008.

VARELLA, M.D. A crescente complexidade do sistema jurídico internacional: alguns problemas de coerência sistêmica. Revista de Informação Legislativa, Brasília, v. 42, n. 167, jul/set. 2005.
WHALLEY, J. Recent regional agreements: why so many, why so much variance in form, why coming so fast, and where are they headed? The World Economy, v. 31, n. 4, 2008.

WORLD BANK. Global economic prospects. Washingon: World Bank, 2005.

WTO. CONSULTATIVE Board to the WTO Director-General, The future of the WTO: advancing institutional challenges in the new Millenium. Geneva: WTO, 2004. 
Para publicar na Revista de Direito Internacional, acesse o endereço eletrônico www.rdi.uniceub.br ou www.brazilianjournal.org.

Observe as normas de publicação, para facilitar e agilizar o trabalho de edição. 